\title{
Measurement of three components in mining and geothermal exploration
}

\author{
I. Ingerov \\ Advanced Geophysical Operations and Services Inc. (AGCOS)
}

\begin{abstract}
In the middle of the last century, surface multiturn coils and quartz variometers served as sensor components of the alternating magnetic field. Later, they were replaced by fluxgate sensors measuring the alternating magnetic field against its constant component and induction sensors reacting only to the variable component. Thanks to a wide frequency range and a low level of generated noise, induction sensors became the main instrument, converting alternating magnetic field into an electric signal. However, there are significant difficulties when it comes to their installation at survey sites, particularly for the vertical component $(\mathrm{Hz})$. This process is first of all, very labour intensive and, second, at times simply impossible due to local conditions, such as rocks, slopes, sand, snow and ice. A solution has been found in utilizing custom designed tripods, which are used not only to accurately align the sensors but to also easily transport them. These are currently mass produced and easily accessible on the market. In recent years, many successful mining geophysics projects have been completed with both geological and economic success in large part due to the use of these tripods. In some cases, these projects were only possible as a result of tripod application since they allow carrying out successful field surveys with AMT, MVP and CSAMT methods on any terrain and soil conditions.
\end{abstract}

Keywords-MVP; three orthogonal components; alternating magnetic field; mining and geothermal exploration

\section{INTRODUCTION}

Magnetovariational profiling method (MVP) was established in in the 1950-60s and widely used for the detection and parameter estimation of large anomalies in the electrical conductivity of the earth's crust and upper mantle ${ }^{[1-}$ 6]. Quartz variometers served as sensors of magnetic components of the EM field, effectively operating in the 10$1000 \mathrm{sec}$ frequency range. This frequency range did not allow for the implementation of the method for resolving mining exploration tasks. The situation changed fundamentally at the turn of the century when 5th generation multifunction equipment appeared on the market, including portable AMT band induction sensors ${ }^{[8-10]}$. 5-channel AMT became widely used in mining exploration. This experience demonstrated that magnetovariational response functions (induction vector and tipper) significantly increase the possibility of conductive objects localization and can be used independently at the exploration stage. This is particularly appealing, since conducting 3-component measurements of the magnetic field does not require instrument grounding, which is desirable when conducting exploration on crystalline shields or during the winter months. However, there is also a considerable difficulty, created by the need to install an induction sensor of the vertical magnetic field component on hard terrain. A fundamental solution to this problem was found in Canada in the first decade of this century. It consists of installing the induction magnetic sensors in custom precision tripods. This allowed the MVP method to become all-season and increased the productivity of works performed using this method by multiple times. In addition, methods of rapid interpretation of the vertical crosssections of the tipper with the purpose of establishing the main parameters of ore objects were proposed at the same time in the works of Ingerov and Ermolin ${ }^{[11]}$. Thus by the end of the first decade of the current century the MVP method became self-sufficient, highly sensitive, and highly productive for mining geophysics ${ }^{[12]}$. This method can be used independently or in conjunction with the AMT method (if local conditions allow for grounding). The MVP method has a well-developed theoretical base ${ }^{[5,6]}$, productive high-precision equipment ${ }^{[10]}$, data processing and interpretation software, and methods of express interpretation ${ }^{[11]}$.

\section{THEORY}

The method's theory was developed in the middle of the last century through the work of Wiese ${ }^{[1]}$, Vozoff ${ }^{[2]}$, Parkinson [3], Schmucker ${ }^{[4]}$, Rokityansky ${ }^{[5,6]}$. Real and imaginary induction vectors, as well as tipper magnitude and phase, were proposed as response functions. Links were established between these functions and the parameters of conductive objects. To date, effective 2-D and 3-D inversion programs of magnetovariational response functions have been developed and methods of express interpretation of vertical tipper cross-sections have been proposed, with the purpose of establishing the parameters of conductive objects.

\section{FIELD EQUIPMENT, INSTRUMENTS AND METHODOLOGY OF FIELD WORK}

To register three orthogonal magnetic components of the earth's natural alternating magnetic field, wide-range of 5th generation multi-function equipment with 3,5 or more recording channels can be used ${ }^{[10]}$. Fig.1,B) shows a layout of a field installation in a 5-channel version. If receiving electric (E) lines are removed, we are left with a standard installation of the MVP method.

A distinguishing feature of this modern field work technology is the fact that all three magnetic field sensors are installed in a three-axis tripod. The tripod has two configurations: the operating position, where the sensors are located orthogonally, oriented to the azimuth, and levelled (Fig.1, A)), and the transportation mode, where sensors are 
parallel. In the latter case, they are easy to transport using a custom frame (Fig. 1, C)). Converting the tripod from

A)

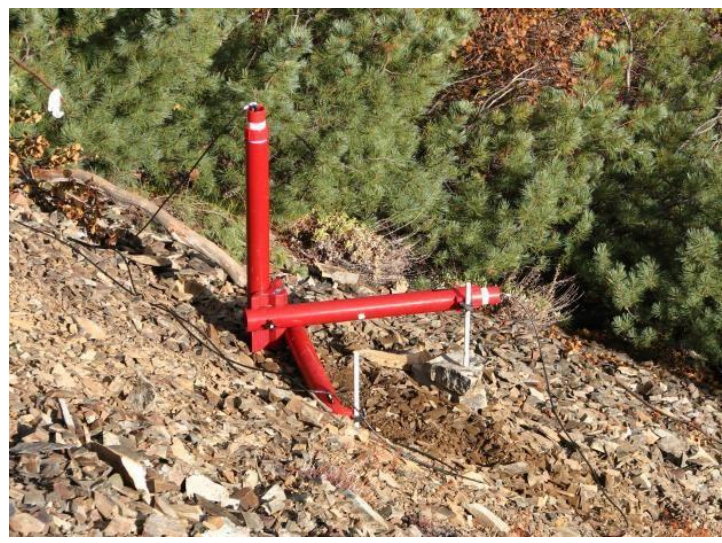

B)



C)

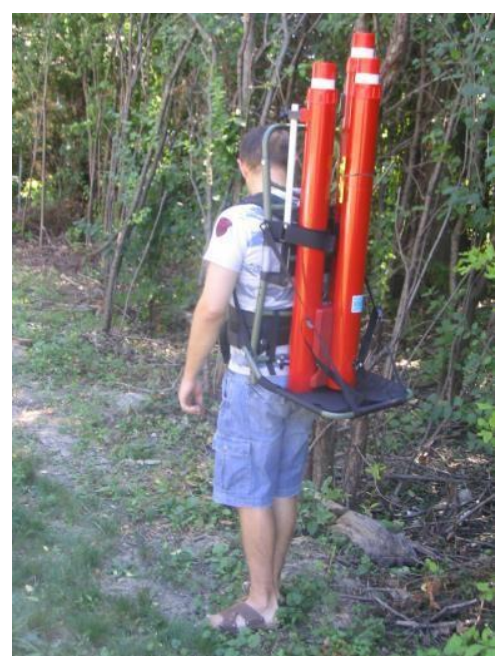

Fig.1 Typical layout of MVP observation site in the field; A) - tripod with magnetic sensors in data record mode; B) - typical 5- component AMT/MVP site layout; C) - tripod in transportation mode.

its transport mode to the operating mode (and vice versa) takes 1-3 min. The use of tripods significantly improves productivity and accuracy of field work, as well as increases the thermal stability of magnetic sensors. In detailed surveys using 4- and 8-channel multi- function devices, tripod application can also improve productivity due to the fact that they allow the field team to record the field on 2-4 sites simultaneously. Data processing is performed with standard programs and does not require any specialized software. However, it is desirable to use programs that allow the use of a remote reference station and the calculation of the magnetic tensor.

\section{METHODS OF EXPRESS INTERPRETATION}

On vertical tipper pseudo-sections over conductive objects, one can observe two maxima anomalies, amplitude and location of which on the vertical pseudo-section is closely tied to the parameters of the body, such as: depth of the centre of the deposit, total longitudinal conductivity of the object, and the form (angle of inclination) of the object. Some of the mentioned factors are shows in Fig.2 and Fig.3. Using analytical or graphic dependency, it is possible over the course of field work, to estimate the location of desirable objects and adjust the course of the field work ${ }^{[11]}$.

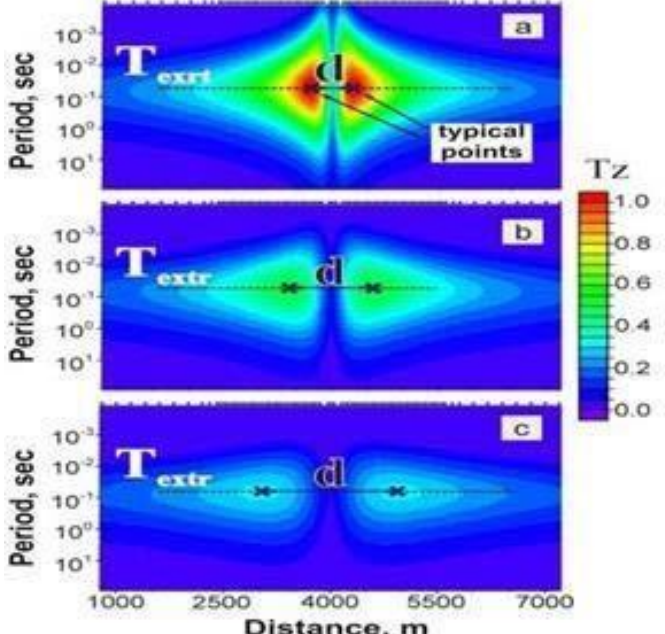

Fig. 2 Dependence of tipper maxima position at pseudo-section from the depth of the center of conductive isometric body; a - $300 \mathrm{~m}, \mathrm{~b}-600 \mathrm{~m}, \mathrm{c}$ $900 \mathrm{~m} ; \mathrm{d}$ is increasing with the depth.
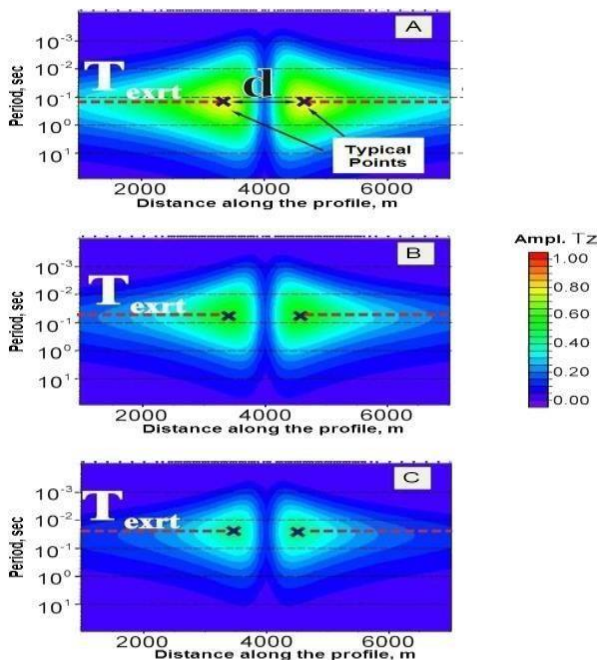

Fig.3 Tipper pseudosection for 2-D conductive bodies with different conductivity of the section. T is increasing with the increase of $\mathrm{d}$.

\section{V.FIELD RESULTS OF COMMERCIAL AND SCIENTIFIC WORKS}

Fig.4 shows the result of field work on the regional profile in Siberia. The work was performed by Alrosa Co. Ltd. (Russia). The goal of the survey was to establish exact 
location of the fault controlling the position of kimberlitic dikes. The top part of the Figure 4 shows real induction vectors according to the Wiese-Schmucker convention (directed away from the conductor). The position of the fault clearly indicated the location of the change in vector direction. Fig.5 shows the results of the MVP survey conducted in the Arkhangelsk region with the goal of locating kimberlitic pipes. The survey was conducted by the Nord-West Ltd. (Moscow, Russia). The Fig.5, B) shows real induction vectors constructed according to the WieseSchmucker convention. The direction and size of the vectors clearly indicate the location of a kimberlitic pipe, which is a more conductive object.

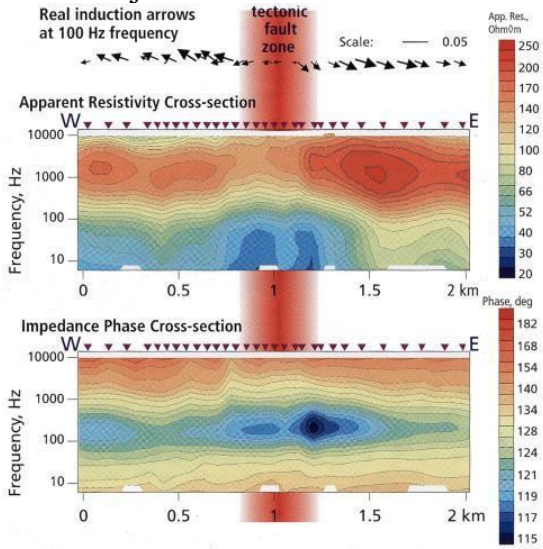

Fig.4 Practical results of MVP method response functions application during mining exploration surveys. Kimberlitic AMT survey for deep fault mapping (Alrosa Co. Ltd., 2004); Induction vectors (Wiese-Schmucker plotting convention) and vertical amplitude and phase resistivity cross-sections above the fault in Yakutiya region (Russia).

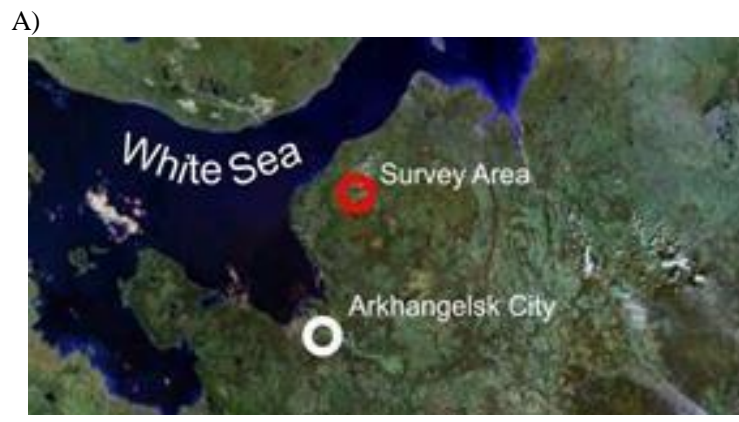

B)

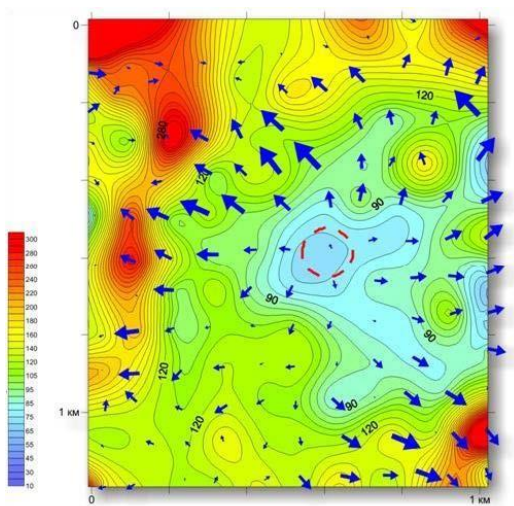

Fig.5. Results of MVP survey; A) - location of the survey area;
B) - apparent resistivity and induction vectors at $500 \mathrm{~Hz}$. Induction vectors (Wiese-Schmucker convention) point to kimberlitic pipe in Arkhangelsk survey (Nord West Ltd., 2005).

\section{CONCLUSIONS}

To date, the MVP method has all the attributes of a successful geophysical method:

- Theory

- Instrument base

- Advanced technology of field work using precision tripods

- Software for data processing, editing and analysis of field data

- Interpretation technology, including 2-D and 3-D inversion methods for rapid determination of parameters of anomalous objects.

\section{REFERENCE}

[1] Wiese, H., 1965, Geomagnetic Tiefentellurik: Deutsche Akademie der Wissenschaften, Geomagnetic Institute Potsdam, Abhandlungen, 36, 1146.

[2] Vozoff, K., 1991, The magnetotelluric method, In: Nabighian, M. (Ed.) Investigations in Geophysics, No.3 - Electromagnetic Methods in Applied Geophysics, Vol.2, Application/Parts A and B, SEG publication.

[3] Parkinson, W.D., 1959, Direction of rapid electromagnetic fluctuation: Geophysical Journal International, 2 (1), 1-14. DOI: 10.1111/j.1365246X.1959.tb057.

[4] Schmucker, U., 1970, Anomalies of geomagnetic variations in the south western United States: Bulletin of the Scripps Institution of Oceanography, University of California Press.

[5] Rokityansky, I.I., 1975, Investigation of electrical conductivity anomalies by the method of magnetovariation profiling: Naukova Dumka.

[6] Rokityansky, I.I., 1982, Geoelectromagnetic investigation of the earth's crust and mantle: Springer-Verlag.

[7] Fox, L., 2008, Fifth generation of multifunctional equipment - ten years in the market, The 19th International Workshop on Electromagnetic Induction in the Earth, Beijing, China, Abstracts, Vol.1, 432-436.

[8] Ingerov, O., et al., 2008, High sensitivity EM prospecting technique based on measurement of three magnetic components of natural EM field, 19th IAGA WG Workshop on Electromagnetic Induction in the Earth, Beijing, 965-970.

[9] Ingerov, O., et al., 2009, Non-grounded Surface Electroprospecting Technique, 71st EAGE Conference \& Exhibition incorporating SPE EUROPEC 2009.

[10] Ingerov, O., 2011, Current trends in the development of electroprospecting hardware set for ground and marine surveys, EMS2011, St. Petersburg, Russia, Abstracts, Vol.1, 86-101.

[11] Ingerov, O., and Ermolin, E., 2010, The parameter estimation of 2-D conductive isometric bodies by singular points at the tipper frequency characteristic, Proceedings of 20th Induction Workshop IAGA, Giza, Egypt-2010, September 18-24, 303-306.

[12] Ermolin, E., et. al., 2011, The Results of AMT Survey at Patomsky Crater, 73rd EAGE Conference \& Exhibitionincorporating SPE EUROPEC 2011 in Vienna, Austria 23- 26, May 2011, 303-306. 